\title{
Aspirin in the era of immunotherapy
}

Editorial

\section{Tsuyoshi Hamada, Marios Giannakis and Shuji Ogino}

Immune checkpoint inhibitors against the $P D C D 1$ (programmed cell death 1, PD-1)-CD274 (PDCD1 ligand 1, PD-L1) axis have shown unprecedented clinical benefits in the treatment of refractory neoplasms [Gentzler R, et al. Immunotherapy. 2016;8:583-600]. Recently, the U.S. Food and Drug Administration (FDA) approved the antiPDCD1 (PD-1) antibody pembrolizumab for treating solid tumors with high-level microsatellite instability (MSI) or mismatch repair deficiency. This approval attracted great attention as the first of an agent based on a tumor biomarker rather than the primary cancer site. Highlevel MSI is commonly present in colorectal carcinomas, and abundant neoantigens due to frequent frameshift mutations have been proposed as a potential explanation of the survival benefits seen with the immune checkpoint blockade in this tumor subtype [1]. However, not all MSIhigh colorectal carcinomas respond to the immunotherapy. The oncogenesis of colon and rectal cancer represents a complex process that is influenced by lifestyle factors, the microbiome, and host cells, potentially leading to not only a unique combination of genetic and epigenetic aberrations but also a distinct microenvironment [2,3]. Therefore, there exists an important need to identify predictive factors for response to immunotherapy beyond tumor MSI status and to develop effective combination treatment strategies.

Aspirin is a common nonsteroidal anti-inflammatory drug (NSAID) that inhibits PTGSI (cyclooxygenase-1) and PTGS2 (cyclooxygenase-2), and regular aspirin use has been shown to reduce colorectal cancer incidence and mortality [Drew DA, et al. Nat Rev Cancer. 2016;16:173186]. Taking the inter-tumor heterogeneity into account, large cohort studies suggest that survival benefits from postdiagnosis aspirin use are pronounced for colorectal cancer with $P T G S 2$ overexpression or activating PIK3CA mutations [4, 5] [Domingo E, et al. J Clin Oncol. 2013:31:4297-4305; Gray RT, et al. Clin Transl Gastroenterol. 2017:8:e91], supporting the potential of these molecular alterations as tumor biomarkers for response to adjuvant aspirin therapy. Notably, these findings provide population-based evidence for the underlying mechanisms by which aspirin may suppress colorectal cancer progression: i.e., aspirin may reduce colorectal cancer mortality by inhibiting PTGS2 and prostaglandin E2 (PGE2) synthesis that are enhanced by activated PI3K signaling. Beyond these potential antitumor effects associated with inhibition of oncogenic signaling pathways, accumulating evidence points to immune-enhancing effects of aspirin on adaptive and innate immune response, including $\mathrm{T}$ cell-mediated antitumor immunity [6].

Recently, we reported a U.S. population-based study which suggested a stronger survival association of postdiagnosis aspirin use in colorectal cancer with lower-level CD274 (PD-L1) expression than in cancer with higher-level CD274 expression (Figure 1) [7]. This study was motivated by experimental data supporting a synergistic effect between aspirin and anti-PDCD1 antibody [8]. In the study by Zelenay et al. [8], PTGS (cyclooxygenase)-induced PGE2 was shown to play a key role in suppressing anti-tumor immune response and promoting growth of cancer cells through increased levels of inflammatory mediators. In a mouse model, PTGS inhibition via aspirin synergized with the immune checkpoint blockade toward immune eradication of

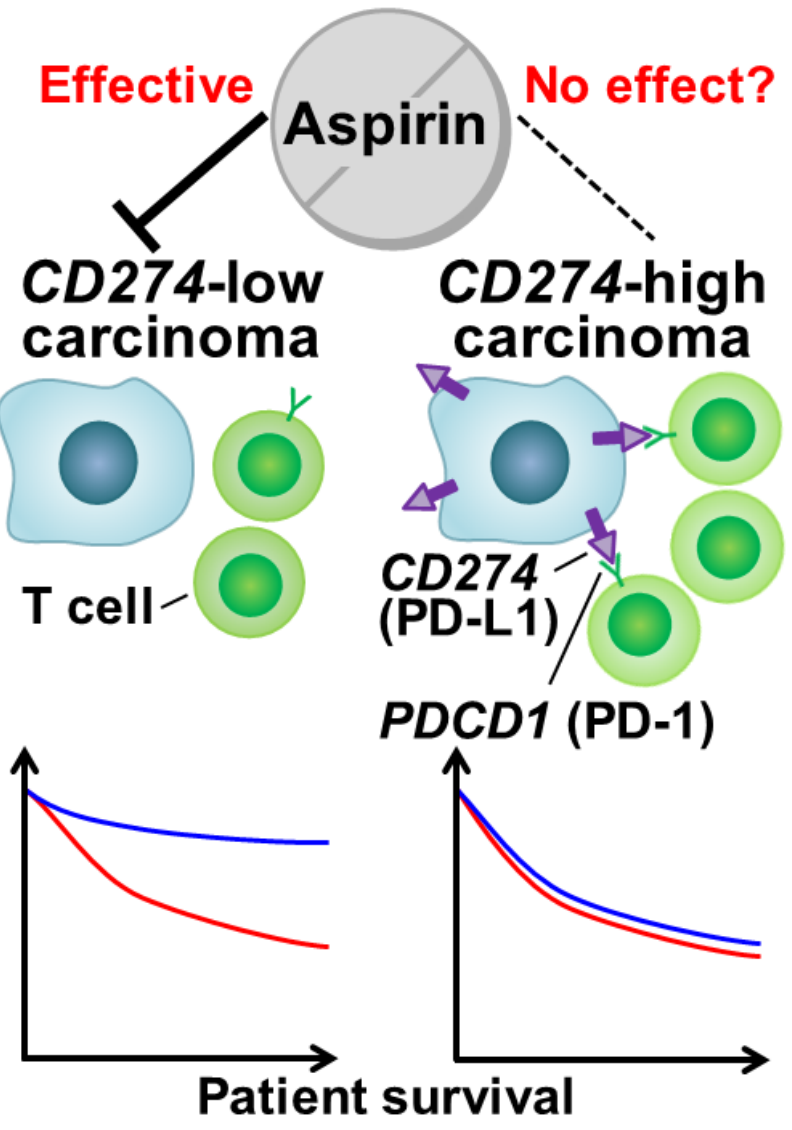

Figure 1: Postdiagnosis aspirin therapy and colorectal cancer progression according to tumor CD274 (PDL1) expression status. 
tumors. Based on these experimental and preclinical findings, we analyzed 617 colon and rectal carcinoma patients from two large prospective studies, and examined the differential survival association of postdiagnosis aspirin use by tumor CD274 expression status, as evaluated by immunohistochemistry [7]. We found significantly lower colorectal cancer-specific mortality associated with postdiagnosis aspirin use in CD274-low tumors, but not in CD274-high tumors $\left(P_{\text {interaction }}<0.001\right)$. This differential survival association was consistently observed regardless of levels of tumor-infiltrating lymphocytes defined by histopathologic examination. These findings indicate that activation of the CD274-PDCD1 immune checkpoint pathway may confer resistance to aspirin therapy, and that this could be overcome with the immune checkpoint blockade. This study was the first population-based study to suggest an interaction between immune checkpoint status and PGE2 inhibition via aspirin in regulating the progression of human colorectal cancer.

These aspirin studies were based on the paradigm of molecular pathological epidemiology (MPE) for survival analyses [2, 3]. In contrast to conventional epidemiology which examines the relationship of an exposure of interest with the outcome of patients grouped into a single disease entity (e.g., colorectal cancer), MPE research classifies the disease by means of tumor molecular and pathologic characterizations, and elucidates differences in treatment outcomes according to patterns of molecular alterations (e.g., CD274-low vs. CD274-high). Furthermore, the aspirin studies utilized the methodology of pharmacoepidemiology, clearly illustrating the successful integration of this traditional discipline with MPE (termed "pharmaco-MPE") [3]. Importantly, these studies highlight the notable strengths of pharmaco-MPE: i.e., pharmacoMPE research can 1) refine the effect estimate of the association of a medication with the outcome of a specific disease subtype, thereby identifying potential biomarkers for clinical benefits; 2) provide evidence on underlying mechanisms of benefits or harms of the medication; and 3) help develop tailored treatment strategies. As is clear from these features of MPE, MPE has emerged and evolved in parallel with the global trend toward precision medicine.

In summary, our study [7] underscores not only the potential role of the immune checkpoint status in modulating survival benefits of adjuvant aspirin therapy for colorectal cancer, but also the potential synergism of aspirin and the immune checkpoint blockade for combination immunotherapy strategies. These findings provide supporting evidence for future clinical studies investigating aspirin therapy in a target population (i.e., patients with CD274-low colorectal cancer) and in those receiving immune checkpoint inhibitors. Given the importance of better understanding of the tumor-immune microenvironment, consideration of immune parameters in MPE research would provide ample opportunities for development of novel immunomodulatory strategies of cancer prevention and treatment (which can be termed “immuno-MPE") [3].

Shuji Ogino: Department of Oncologic Pathology, DanaFarber Cancer Institute and Harvard Medical School, Program in MPE Molecular Pathological Epidemiology, Department of Pathology, Brigham and Women's Hospital and Harvard Medical School and Department of Epidemiology, Harvard T.H. Chan School of Public Health, Boston, MA, USA

Correspondence to: Shuji Ogino, email shuji_ogino@dfci. harvard.edu

Keywords: clinical outcome, colorectal neoplasms, molecular pathological epidemiology, non-steroidal anti-inflammatory drugs, tumor microenvironment

Received: August 31, 2017

Published: September 14, 2017

\section{REFERENCES}

1. Le DT, et al. Science. 2017; 357: 409-413.

2. Ogino S, et al. Gut. 2011; 60: 397-411.

3. Ogino S, et al. Epidemiology. 2016; 27: 602-611.

4. Liao X, et al. N Engl J Med. 2012; 367: 1596-1606.

5. Chan AT, et al. JAMA. 2009; 302: 649-658

6. Zhang Q, et al. Front Immunol. 2017; 8: 71.

7. Hamada T, et al. J Clin Oncol. 2017; 35: 1836-1844.

8. Zelenay S, et al. Cell. 2015; 162: 1257-1270.

Copyright: Hamada et al. This is an open-access article distributed under the terms of the Creative Commons Attribution License 3.0 (CC BY 3.0), which permits unrestricted use, distribution, and reproduction in any medium, provided the original author and source are credited. 